\title{
Transcriptomic profiling to identify genes involved in Fusarium mycotoxin Deoxynivalenol and Zearalenone tolerance in the mycoparasitic fungus Clonostachys rosea
}

Chatchai Kosawang ${ }^{1,2^{*}+}$, Magnus Karlsson ${ }^{2+}$, Dan Funck Jensen ${ }^{2}$, Adiphol Dilokpimol ${ }^{1}$ and David B Collinge ${ }^{1}$

\begin{abstract}
Background: Clonostachys rosea strain IK726 is a mycoparasitic fungus capable of controlling mycotoxin-producing Fusarium species, including F. graminearum and F. culmorum, known to produce Zearalenone (ZEA) and Deoxynivalenol (DON). DON is a type B trichothecene known to interfere with protein synthesis in eukaryotes. ZEA is a estrogenic-mimicing mycotoxin that exhibits antifungal growth. C. rosea produces the enzyme zearalenone hydrolase (ZHD101), which degrades ZEA. However, the molecular basis of resistance to DON in C. rosea is not understood. We have exploited a genome-wide transcriptomic approach to identify genes induced by DON and ZEA in order to investigate the molecular basis of mycotoxin resistance C. rosea.
\end{abstract}

Results: We generated DON- and ZEA-induced CDNA libraries based on suppression subtractive hybridization. A total of 443 and 446 sequenced clones (corresponding to 58 and 65 genes) from the DON- and ZEA-induced library, respectively, were analysed. DON-induced transcripts represented genes encoding metabolic enzymes such as cytochrome P450, cytochrome c oxidase and stress response proteins. In contrast, transcripts encoding the ZEA-detoxifying enzyme ZHD101 and those encoding a number of ATP-Binding Cassette (ABC) transporter transcripts were highly frequent in the ZEA-induced library. Subsequent bioinformatics analysis predicted that all transcripts with similarity to $A B C$ transporters could be ascribed to only $2 A B C$ transporters genes, and phylogenetic analysis of the predicted $A B C$ transporters suggested that they belong to group $G$ (pleiotropic drug transporters) of the fungal $A B C$ transporter gene family. This is the first report suggesting involvement of $A B C$ transporters in ZEA tolerance. Expression patterns of a selected set of DON- and ZEA-induced genes were validated by the use of quantitative RT-PCR after exposure to the toxins. The qRT-PCR results obtained confirm the expression patterns suggested from the EST redundancy data.

Conclusion: The present study identifies a number of transcripts encoding proteins that are potentially involved in conferring resistance to DON and ZEA in the mycoparasitic fungus C. rosea. Whilst metabolic readjustment is potentially the key to withstanding DON, the fungus produces ZHD101 to detoxify ZEA and ABC transporters to transport ZEA or its degradation products out from the fungal cell.

Keywords: Clonostachys rosea, cDNA library, Fusarium mycotoxins, Deoxynivalenol, Zearalenone

\footnotetext{
*Correspondence: chatchai.kosawang@gmail.com

${ }^{\dagger}$ Equal contributors

'Department of Plant and Environmental Sciences, University of Copenhagen, Copenhagen, Denmark

${ }^{2}$ Uppsala BioCenter, Department of Forest Mycology and Plant Pathology,

Swedish University of Agricultural Sciences, Uppsala, Sweden
} 


\section{Background}

The Fusarium head blight disease of cereals is caused by members of the Fusarium species complex, including $F$. graminearum, F. culmorum, F. avenaceum and F. poae [1]. These Fusarium spp. are well known for their ability to produce a plethora of secondary metabolites, some of which act as mycotoxins since they possess the ability to affect animals and humans adversely. Deoxynivalenol (DON) and Zearalenone (ZEA) are among the most predominant mycotoxins commonly found in infected seeds and grains [2]. DON belongs to the type B group of trichothecenes and is produced ubiquitously during plant infection where it can act as a virulence factor [3]. DON is a potent protein synthesis inhibitor which binds eukaryotic ribosomes and hampers protein translation $[4,5]$. DON repressed the activity of the cell wall degrading enzyme $\mathrm{N}$-acetyl-beta-D-glucosaminidase in the biocontrol fungus Trichoderma atroviride, proposing an additional role of DON in Fusarium competitiveness besides being a disease virulence factor [6]. ZEA is a non-steroidal mycoestrogenic toxin that is produced largely by F. graminearum, F. culmorum and F. equiseti [7]. The molecular structure of ZEA resembles that of the mammalian hormone $17 \beta$-estradiol, thus consumption of the toxin by mammals stimulates hypoestrogenic responses and can result in infertility and has also been linked to cancer $[4,8]$. Limited information about the biological roles of ZEA is available, although it has been speculated that ZEA has functions in binding and activation of the $\mathrm{K}^{+}$channel $\beta$ subunit, involved in a signal transduction pathway [9]. ZEA has been shown to possess antifungal properties propounding the hypothesis that ZEA is synthesized to increase competitiveness with other fungi inhabiting the same niche [10].

Microbial detoxification of DON and ZEA has been observed by various organisms and distinct mechanisms are involved. For example, Aspergillus spp. disarmed the toxic effects of ZEA by conversion of the toxin to zearalenone-sulphate [11]. The yeast Trichosporon mycotoxinivorans was proposed to transform ZEA by cleaving a lactone ring backbone in the similar way to the detoxifying mechanism described from Clonostachys rosea that relies on action of the enzyme zearalenone hydrolase (EC 3.1.1.-; alternative: zearalenone lactonase, zearalenone lactonohydrolase; ZHD101) [12-14]. A recent finding reported an ability to degrade ZEA in the bacterium Rhodococcus pyridinivorans, although the detoxification mechanism of the strain is not yet known [15]. On the other hand, peroxidase was speculated to degrade DON in A. oryzae and Rhizopus oryzae [16] whereas hydrolytic breakdown of DON was found in A. tubingensis [17]. Several bacteria have also been found to be capable of detoxifying DON. These included a Bacillus sp., Lactobacillus pentosus, L. paracasei and L. plantarum [18]. Thus, de- epoxidation, epimerization and mineralization are reviewed as three important DON detoxifying mechanisms in microorganisms [19-21].

Clonostachys rosea (Teleomorph: Bionectria ochroleuca) is an ascomycete fungus with a wide range of lifestyles. The fungus has been reported to live as a saprophyte, as a nematophagous fungus and as a mycoparasitic fungus $[22,23]$. The C. rosea strain IK726 is a mycoparasitic fungus that is effective in controlling plant pathogens, including Alternaria spp. - the causative agent of black rot of carrot, Bipolaris sorokiana - the causative agent of spot blotch of barley and DON- and ZEA-producing F. culmorum [22-24]. Despite showing tolerance to DON, the underlying mechanism in the fungus remains obscure. In this study, we aimed to understand mechanisms regulating resistance to DON and to investigate whether additional mechanisms are involved in resistance to ZEA besides the well-known ZHD101 in C. rosea. We generated cDNA libraries enriched in genes expressed during interaction with DON and ZEA. Induction of genes by DON and ZEA were subsequently confirmed by the use of quantitative reverse transcription real-time PCR (qRT-PCR). Analysis of our functional annotation data suggests that metabolic readjustment is a major component for DON tolerance and ATP- Binding Cassette (ABC) transporters are involved in providing tolerance to $\mathrm{ZEA}$, in addition to ZHD101.

\section{Results}

\section{Construction of DON- and ZEA-induced CDNA libraries}

Of 480 sequenced clones from the DON- and ZEAinduced libraries, 443 and 446 high-quality ESTs were obtained after sequence cleansing, respectively. BLASTX of the DON-induced 443 ESTs yielded 230 unigene EST sets representing 161 genes with similarity to characterized proteins (E-value $\leq 10^{-6}$ ), 82 ESTs with hypothetical proteins and 131 transcripts with either no similarity to known sequences (e-value $\geq 10^{-6}$ ). The 446 high quality ESTs from the ZEA library represented 334 unigene EST sets, BLAST searches returned 412 ESTs with similarity to characterized proteins, 4 ESTs matched hypothetical proteins and 30 ESTs showed no similarity.

Up to $92 \%$ of the ESTs with similarity to characterized proteins from the DON-induced library were assigned a GO functional annotation. The major clusters were metabolic process and cellular process which represented $33 \%$ and $31 \%$ of the total ESTs, respectively. Similarly, analysis of 396 ZEA-induced ESTs with similarity to characterized proteins showed that metabolic process was the largest cluster accounting for 39\% of the total ESTs followed by cellular process and localization at $33 \%$ and $21 \%$, respectively. Distribution of ESTs from each library based on putative functional annotation is shown in Figure 1. The ESTs from DON- and ZEA- 


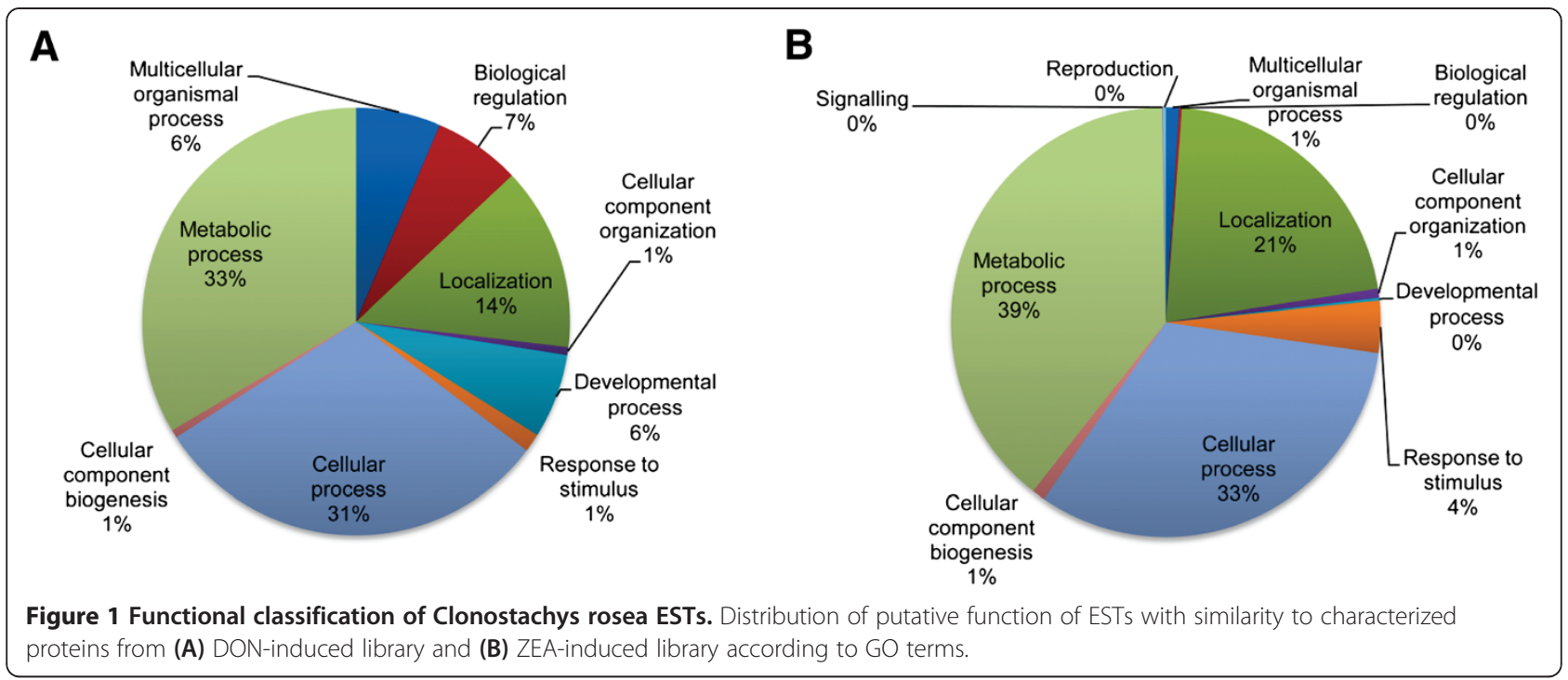

induced libraries were deposited at DNA Data Bank of Japan (DDBJ) under accession nos. FY998777-FY998944 and FY998945-FY999086, respectively. The ESTs that were present in high redundancy ( $\geq 4$ times) are listed in Tables 1 and 2 for DON- and ZEA-induced libraries, respectively. Complete putative annotation and best BLAST hit are presented in Additional files 1 and 2.
Highly redundant $C$. rosea genes in the DON-induced library

A set of transcripts induced by DON were classified with putative functions in metabolism, cell cycle, transport and stress response. The majority of the redundant transcripts putatively encoded metabolic or biosynthetic enzymes, for instance, 7 of cytochrome P450 55A3 (CYP450 55A3;

Table 1 Transcripts that present in high frequency in DON-induced CDNA library

\begin{tabular}{|c|c|c|c|c|}
\hline Annotated transcripts & E-value & Count & Organism & DDBJ accession \\
\hline \multicolumn{5}{|l|}{ Metabolism } \\
\hline ATP synthase alpha chain mitochondria precursor & $2 e^{-64}$ & 14 & Sclerotinia sclerotiorum & FY998859 \\
\hline Cytochrome C oxisase polypeptide VIb & $7 e^{-43}$ & 12 & Trichoderma reesei & FY998809 \\
\hline Acyl-CoA desaturase & $2 e^{-136}$ & 9 & Gibberella zeae & FY9988663 \\
\hline Cytochrome P450 55A3 & $5 e^{-122}$ & 7 & Fusarium lichenicola & FY998833 \\
\hline Alcohol dehydrogenase & $9 e^{-109}$ & 6 & Trichoderma virens & FY998868 \\
\hline Glycoside hydrolase family 76 protein & $3 e^{-43}$ & 6 & Trichoderma virens & FY998816 \\
\hline Diacylglycerol o-acyltransferase $2 \mathrm{~b}$ & $1 e^{-103}$ & 5 & Trichoderma reesei & FY998849 \\
\hline \multicolumn{5}{|l|}{ Transport } \\
\hline Plasma membrane ATPase ( $\mathrm{H}^{+}$-ATPase) & $9 e^{-80}$ & 22 & Trichoderma reesei & FY998804 \\
\hline High affinity glucose transporter SNF3 & $1 e^{-16}$ & 11 & Verticillium dahlia & FY9988819 \\
\hline Hexose transporter-like protein (TrHXT2) & $2 e^{-141}$ & 6 & Trichoderma reesei & FY9988842 \\
\hline \multicolumn{5}{|l|}{ Stress response } \\
\hline Major allergen asp f2-like protein & $7 e^{-23}$ & 6 & Metarhizium anisopliae & FY998813 \\
\hline Mitochondrial hypoxia responsive protein & $7 e^{-45}$ & 6 & Trichoderma atroviride & FY9988834 \\
\hline \multicolumn{5}{|l|}{ Cell cycle } \\
\hline ThiJ/Pfpl family protein & $1 e^{-131}$ & 29 & Metarhizium acridum & FY998826 \\
\hline CHK1 checkpoint-like protein & $2 e^{-16}$ & 9 & Trametes versicolor & FY998823 \\
\hline Eukaryotic initiation factor 1 SUI1 & $4 e^{-59}$ & 8 & Gibberella zeae & FY998855 \\
\hline Glucose repressible protein grg1 & $7 e^{-29}$ & 5 & Beauveria bassiana & FY998852 \\
\hline
\end{tabular}


Table 2 Transcripts that present in high frequency in ZEA-induced cDNA library

\begin{tabular}{|c|c|c|c|c|}
\hline Annotated transcripts & E-value & Count & Organism & DDBJ accession \\
\hline \multicolumn{5}{|l|}{ Metabolism } \\
\hline Zearalenone hydrolase & $1 e^{-176}$ & 120 & Bionectria ochroleuca & FY998945 \\
\hline Glycoside hydrolase family 5 & $4 e^{-125}$ & 21 & Trichoderma virens & FY998957 \\
\hline Amidophosphoribosyltransferase & $9 e^{-65}$ & 15 & Magnaporthe oryzae & FY998962 \\
\hline Cytochrome P450 & $1 e^{-62}$ & 8 & Trichophyton verrucosum & FY998953 \\
\hline Dihydrolipoyl dehydrogenase & $3 e^{-32}$ & 5 & Metarhizium anisopliae & FY9989666 \\
\hline 4-aminobutyrate aminotransferase & $3 e^{-16}$ & 5 & Verticillium dahlia & FY998971 \\
\hline Pyruvate decarboxylase & $9 e^{-50}$ & 4 & Trichoderma reesei & FY998999 \\
\hline \multicolumn{5}{|l|}{ Transport } \\
\hline ABC transporter CDR4 & $5 e^{-74}$ & 21 & Neurospora crassa & FY999079 \\
\hline multidrug resistance protein CDR1 & $4 e^{-93}$ & 19 & Colletotrichum higginsianum & FY999081 \\
\hline $\mathrm{ABC}$ transporter & $4 e^{-33}$ & 18 & Gibberella pulicaris & FY999076 \\
\hline $\mathrm{ABC}$ transporter & $8 e^{-104}$ & 13 & Nectria haematococca & FY999078 \\
\hline multidrug resistance protein CDR1 & $2 e^{-52}$ & 8 & Colletotrichum higginsianum & FY9990084 \\
\hline $\mathrm{ABC}$ transporter & $8 e^{-33}$ & 8 & Gibberella pulicaris & FY999082 \\
\hline ABC transporter & $1 e^{-113}$ & 6 & Nectria haematococca & FY9990077 \\
\hline pleiotropic drug resistance protein $\mathrm{TABC} 2$ & $7 e^{-32}$ & 6 & Trichoderma atroviride & FY9990083 \\
\hline Bacteriorhodopsin & $6 e^{-14}$ & 6 & Colletotrichum higginsianum & FY998978 \\
\hline Major facilitator superfamily transporter & $8 e^{-39}$ & 5 & Glomerella graminicola & FY998969 \\
\hline Vascuolar protein sorting 26 & $9 e^{-49}$ & 5 & Metarhizium anisopliae & FY9990008 \\
\hline Allantoate permease of major facilitator superfamily & $2 e^{-38}$ & 5 & Glomerella graminicola & FY999011 \\
\hline Mitochondrial phosphate carrier protein & $2 e^{-28}$ & 4 & Trichoderma atroviride & FY9990030 \\
\hline Protein CCC1 & $1 e^{-16}$ & 4 & Metarhizium anisopliae & FY999002 \\
\hline Pleiotropic Drug Resistance family protein & $1 e^{-72}$ & 4 & Trichoderma reesei & FY999085 \\
\hline \multicolumn{5}{|l|}{ Stress response } \\
\hline Heat shock protein 70 & $2 e^{-39}$ & 8 & Nicotiana tabacum & FY9989883 \\
\hline \multicolumn{5}{|l|}{ Cell cycle } \\
\hline FACT complex subunit pop3 & $4 e^{-47}$ & 7 & Nectria haematococca & FY998996 \\
\hline GTP binding protein ychF & $7 e^{-142}$ & 5 & Metarhizium acridum & FY998981 \\
\hline Prohibitin phb1 & $6 e^{-147}$ & 4 & Metarhizium anisopliae & FY998992 \\
\hline
\end{tabular}

EC:1.14.-.-), 12 of cytochrome $\mathrm{C}$ oxidase subunit Vib (COX; EC:1.9.3.1), 5 of diacylglycerol o-acyltransferase (EC:2.3.1.20), 9 of acyl-CoA desaturase (EC:1.14.19.1), and 6 of glycoside hydrolase family 76 (GH76; EC:3.2.1.-).

Other redundant transcripts putatively encoded proteins involved in the cell cycle. ThiJ/PfpI protein family was among the most highly induced ESTs in the DON-induced library, being found 29 times. In addition, ESTs encoding high affinity glucose transporter SNF3, hexose transporterlike protein and plasma membrane ATPase $\left(\mathrm{H}^{+}\right.$-ATPase; EC:3.6.3.6) exhibited increased in expression. We also observed high redundancy for ESTs encoding proteins associated with stress responses. These included molecular chaperones heat shock protein HSP70 and HSP90, mitochondria hypoxia responsive domain protein and flavohemoglobin.

\section{Highly redundant $C$. rosea genes in the}

\section{ZEA-induced library}

Analysis of the ZEA-induced library revealed that the majority of transcripts with high redundancy encoded ZHD101 and $\mathrm{ABC}$ transporters resembling Candida Drug Resistance (CDR) 1 and CDR4 of Candida albicans and ABC-2 type transporters. In addition to ZHD101, ESTs putatively encoding other metabolic enzymes were recorded including CYP450 and amidophosphoribosyltransferase (EC:2.4.2.14). ESTs putatively encoding enzymes involved in glycolysis and TCA such as pyruvate kinase (EC:2.7.1.40), aconitrate hydratase (EC:4.2.1.3) and pyruvate decarboxylase (EC:4.1.1.1) were also present in high numbers in the ZEA-library.

In addition, we found ESTs encoding glycoside hydrolase family 5 (GH5) that exhibits broad known activities, 
including glucan $\beta$-1,3-glucosidase (EC: 3.2.1.58), $\beta$-mannosidase (EC: 3.2.1.25) and chitosanase (EC:3.2.1.132), and other ESTs encoding proteins regulating the cell cycle, e.g., Facilitating Chromatin Transcription (FACT) complex subunit pob3, GTP binding protein (GTPase) $y c h F$ and prohibitin presented in high redundancy in the ZEA-induced library. We also noted transcripts encoding Major Facilitator Superfamily (MFS) transporter induced by ZEA.

\section{Phylogenetic analysis of $A B C$ transporters detected in the ZEA-induced library}

Local BLAST searches to the draft C. rosea IK726 genome sequence revealed that all ESTs from the ZEA-induced library exhibited similarity to only two different $A B C$ transporter genes. The bioinformatic tool FGENESH + was further employed to predict two full-length $A B C$ transporters with 1436 and 1321 amino acids, respectively. Full-length nucleotide sequences of the two predicted genes were shown in Additional file 3. We performed phylogenetic analysis to investigate whether the identified $A B C$ transporters pertain to xenobiotic-transport classes of $\mathrm{ABC}$ proteins. The analysis revealed that they belong to group $\mathrm{G}$ of fungal $\mathrm{ABC}$ transporters (Figure 2) [25] and the two genes were named $a b c G 5$ and $a b c G 29$ according to the nomenclature for fungal $\mathrm{ABC}$ transporters [25].
Group G consists of 7 different subgroups in which most of them harbour relevant functions to either xenobiotic or drug transport. Our analysis indicated that the ABCG5 belong to subgroup I which is related to multidrug resistance, whereas ABCG29 belong to subgroup V which contains members of unknown function.

\section{Gene expression of selected genes from the DON- and ZEA-induced libraries}

To validate genes induced by DON and ZEA, we performed qRT-PCR on 5 selected genes from each library at 2, 6, 12, 36 and 72 hours after inoculation. This temporal gene expression set-up would allow us to monitor the expression dynamics of the candidate genes. In this study, we chose expression of the candidate genes at 2 hours as the calibration point as we foresaw an immediate response of the fungus to ZEA. Analysis with qRT-PCR showed that all the selected genes exhibited a rapid response to both DON and ZEA (Figure 3). After 2-hour exposure to ZEA, the expression of transcripts encoding ZHD101 and ABCG29, identified in the ZEA-induced library, accumulated sharply to more than a thousand fold $(p \leq 0.05)$. Another ABC transporter encoded by $a b c G 5$ was induced 186 -fold $(p \leq 0.05)$. On the other hand, the selected transcripts from the DON-induced library were induced to a

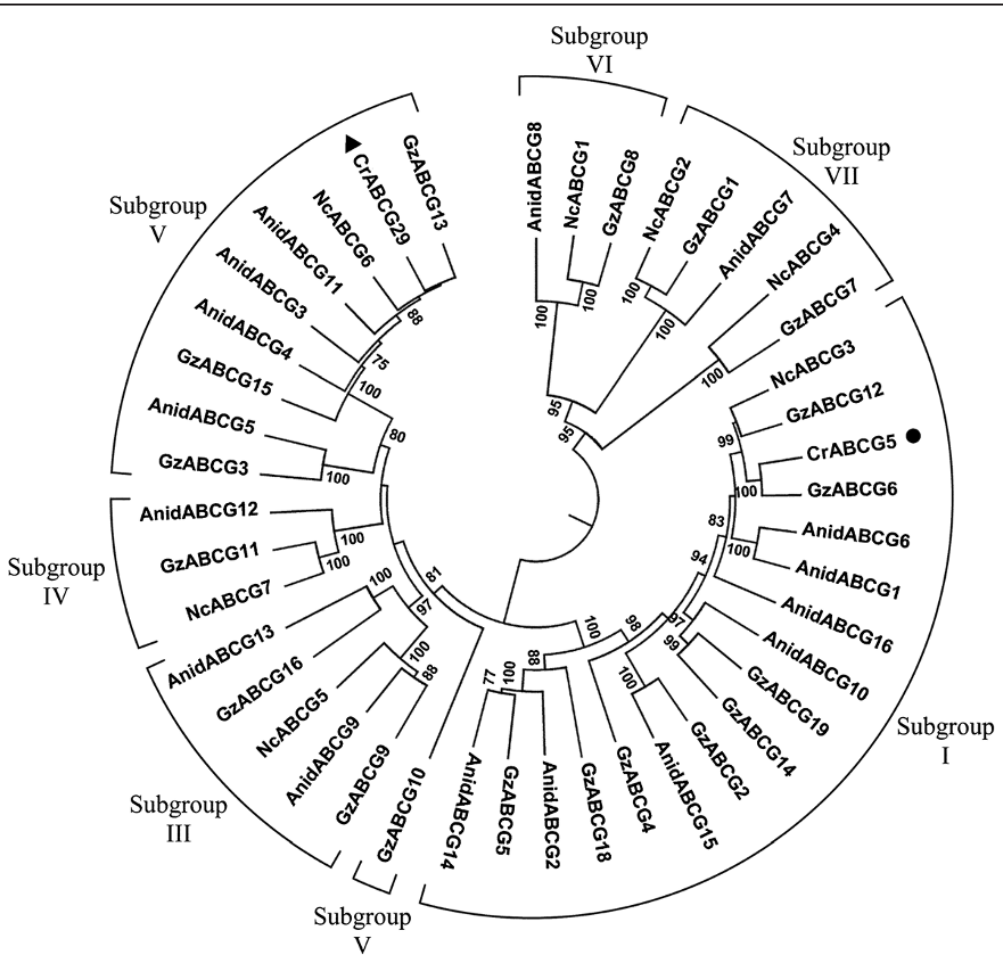

Figure 2 Phylogenetic analysis of fungal subgroup G ABC-transporters. The displayed tree showed only the clade where the two predicted genes - ABCG29 (closed triangle) and ABCG5 (closed circle) - were clustered. ABC-G subgroups were designated according to [25]. Other ABC transporters which were included to generate the tree were from Aspergillus nidulans (Anid), Gibberella zeae (Gz) and Neurospora crassa (Nc). Bootstrap support values ( $\geq 70 \%)$ are associated with branches. 


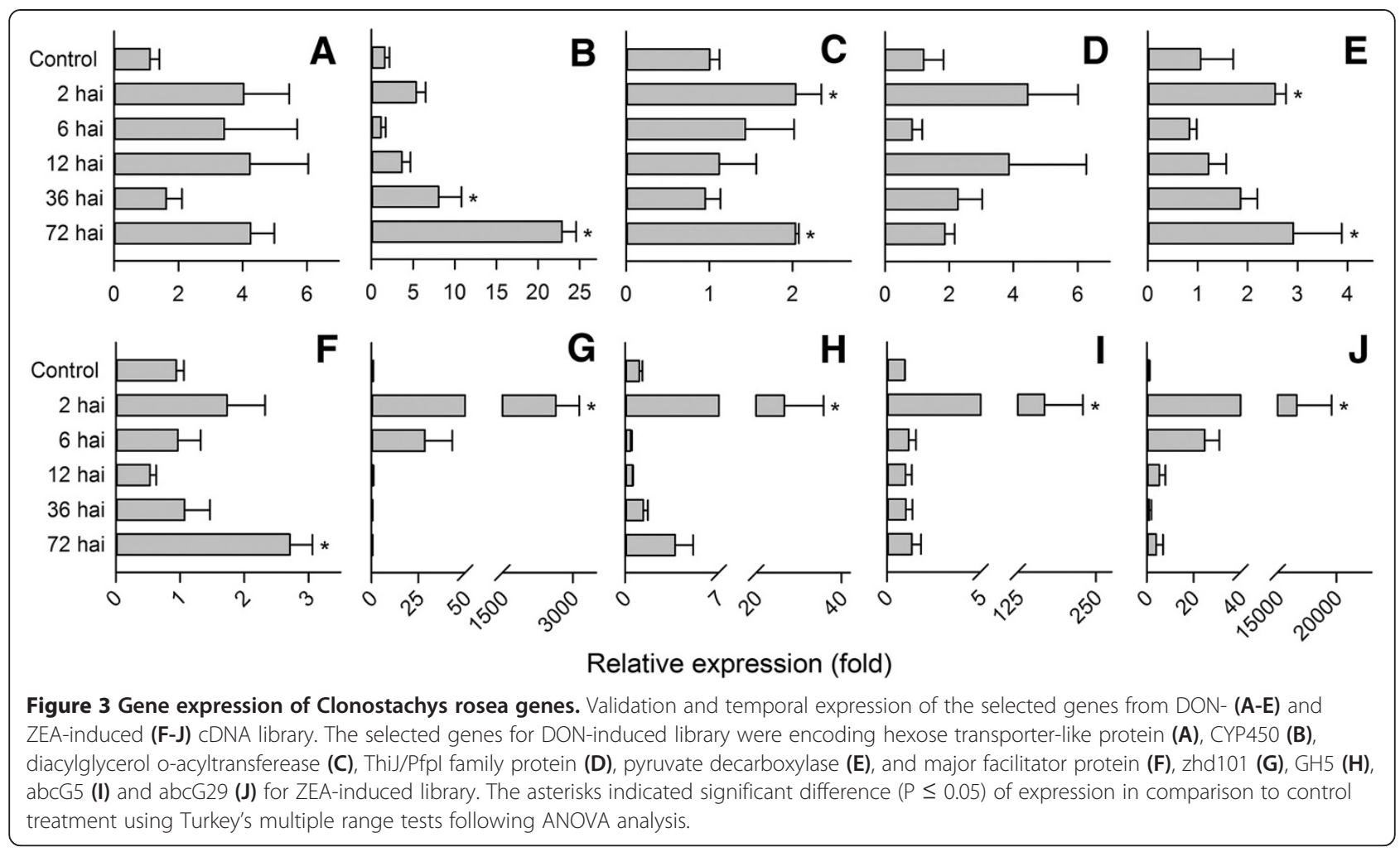

lesser extent. Up to 3- and 2-fold change in expression were detected for pycruvate decarboxylase $(p \leq 0.05)$ and diacylglycerol o-acyltransferease after 2-hour exposure to DON, respectively. Temporal gene expression revealed a substantial drop in expression of most selected genes from ZEA-induced library after 6 hours of exposure whereas 3 of 5 selected genes from DON-induced library were increased in expression at 72 hours (Figure 3). The DONinduced genes with significant alteration in expression $(p \leq 0.05)$ at 72 hours included CYP450 (22 fold), diacylglycerol o-acyltransferease ( 2 fold) and pyruvate decarboxylase ( 2.8 fold).

\section{Discussion}

Tolerance to xenobiotics is of importance for antagonistic fungi during interactions with plant pathogens that produce a broad range of secondary metabolites. Our aim with the current study is to advance our understanding of mechanisms conferring resistance to Fusarium mycotoxins DON and ZEA in the hyperparasitic fungus C. rosea IK726 that is effective in controlling Fusarium species. For this, we employed a genome-wide transcriptomic approach based on suppression subtractive hybridization to explore molecular responses of the fungus towards DON and to ZEA. The analysis of the DON-induced transcripts does not suggest additional mechanisms compared to those previously discovered to render tolerance to DON in microorganisms [19]. This suggests to us that resistance to DON in C. rosea is complex and is the result of synergistic action of proteins from different pathways rather than a standalone mechanism. The analysis of ZEA-induced transcripts suggest that thatZHD101, previously reported as a key enzyme regulating resistance to ZEA in C. rosea, and 2 $\mathrm{ABC}$ transporters may be involved in ZEA resistance.

Metabolic readjustment may be a major component in DON tolerance in C. rosea, as transcripts encoding metabolic enzymes such as CYP450 55A3, COX and mitochondrial ATP synthase are identified in high frequency. Involvement of these enzymes in abiotic stress tolerance has been reported previously. For example, overexpression of COX improved resistance to the antimicrobial peptide MiAMP1 in Saccharomyces cerevisiae [26]. A membrane associated ATP synthase is highly induced in a Cercosporin-resistant Cercospora nicotianae strain but not in a susceptible strain [27].

Exposure to DON induced expression of transcripts encoding a number of transporters. These included the high affinity glucose transporter SNF3, the hexose transporterlike protein TrHXT2 and a plasma membrane $\mathrm{H}^{+}$-ATPase. In $S$. cerevisiae, SNF3 is a glucose sensor that generates a intracellular glucose signalling cascade required for induction of hexose transporter expression, whereas HXT1 (orthologous to TrHXT2) is a high-affinity glucose and mannose transporter [28]. The presence of ESTs encoding proteins similar to SNF3 and TrHXT2 suggests that the demand of cellular energy is increased during DON 
exposure. Taken together with up-regulation of genes encoding metabolic enzymes as mentioned above, it is possible that the increased need of cellular energy is to produce proteins to compensate those destroyed by DON. This idea is supported by the up-regulation of a gene that putatively encodes a proton transporter $\mathrm{H}^{+}$-ATPase, which is shown to facilitate the uptake of nutrients by providing proton gradients for membrane transporters, and to regulate intracellular $\mathrm{pH}$ [29-31]. Interestingly, we also observed the accumulation of transcripts putatively encoding enzymes in the triglyceride synthesis pathway. Triglycerides may act as an energy reservoir and the specific induction of by DON, but not by ZEA, provide further support for an increased energy demand during DON exposure.

DON has been shown to generate a substantial level of reactive oxygen species (ROS) and oxidative stress, which can induce protein damage and DNA strand breakage in human HepG2 cells [32]. This might explain the upregulation of genes encoding stress-response proteins such as the chaperones (heat shock proteins, Hsp) 70 and Hsp90 subunit that possess several important cytoprotective functions, including prevention of protein aggregation and degradation of unstable proteins [33], and the cell cycle checkpoint protein (Chk1) that is essential for cellular function in response to DNA damage [34,35]. Hsp70 and Hsp90 transcripts often accumulate following exposure to biotic and abiotic stresses in several organisms [33,36,37]. As DON generates oxidative stress that damages proteins and DNA, it is likely that Chk1is triggered to protect $C$. rosea from DNA damage by the toxin, while the Hsp70/90 subunits act to protect or recycle damaged proteins.

A previous study showed that alterations in cell wall structure are connected with increased resistance to a killer toxin in S. cerevisiae [38]. DON-exposure induce genes that putatively encode a GH76 and a GH22 enzyme, with known $\alpha-1,6$-mannanase (EC 3.2.1.101) and dolicholP-mannose $\alpha$-mannosyltransferase (EC 2.4.1.-) activity, respectively. These activities are reported to be involved in incorporation of glycoproteins into the cell wall of $N$. crassa and S. cerevisiae $[39,40]$. ZEA-exposure on the other hand induces a putative endoglucanase (EC 3.2.1.-) GH5 gene that is involved in cell wall modification in A. nidulans [41]. We may therefore hypothesise that cell wall modifications are part of the resistance machinery to both DON and ZEA toxins in C. rosea.

ZEA tolerance in C. rosea was determined by ZHD101 that cleaves off one of the lactone rings in the backbone, resulting in the product 1-(3,5-dihydroxyphenyl)-10'hydroxy-1-undecen-6' ${ }^{\prime}$-one, and that the structural change resulted in reduced toxicity of ZEA [12]. In our study, qRT-PCR analysis showed that the fungus responded to ZEA as early as 2 hai and expression of zhd101 decreased significantly at least 200 fold when measured at later time points. This confirms that the time point chosen for library construction was accurate and also supports the previous finding of ZHD101 as a player in ZEA tolerance. Surprisingly, we noticed an increase in expression of transcripts encoding the molecular chaperone Hsp70 in the ZEAinduced library. The fact that the fungus was stressed during exposure to ZEA, despite possessing ZHD101, suggests that ZHD101 is not the only mechanism underlying resistance to ZEA in C. rosea.

$\mathrm{ABC}$ transporters are major secondary transport systems that render resistance to xenobiotics in organisms [25,42]. We hypothesise from the abundant ESTs encoding $A B C$ transporters in the ZEA-induced library that $\mathrm{ABC}$ transporters - together with ZHD101 - contribute to resistance to ZEA in $C$. rosea through the significant increase in transcript levels for $A B C$ transporters from group $G$ (ABC-G) of fungal $A B C$ transporters, which are well known for contributing to drug/fungicide resistance in many fungi [25]. This is supported by the concomitant expression of the two ABC-G proteins have with ZHD101. This is the first report to suggest that the $A B C$ transporters are potentially involved in providing resistance to ZEA. Previously, Kakeya and colleagues demonstrated that the product of ZHD101 activity on ZEA, namely 1-(3,5dihydroxyphenyl)-10' -hydroxy-1-undecen-6' -one, did not possess any estrogenic potencies to human breast cancer MCF-7 cells [12]. Nonetheless, it is unclear whether this degradation product 1-(3,5-dihydroxyphenyl)-6'hydroxy-1-undecen-10'-one possesses toxic activities and thus it triggers expression of the $\mathrm{ABC}$ transporters or whether the $A B C$ proteins act as ZEA efflux pump preventing cells from being damaged from ZEA when ZHD101 is degrading the toxin.

Group $\mathrm{G}$ of fungal ABC transporters comprises 5 subfamilies [25], and our phylogenetic analysis of the predicted full-length $C$. rosea $\mathrm{ABC}$ transporters suggested that the transporters belong to the subgroup I (ABCG5) and subgroup V (ABCG29) of subfamily G. The subgroup I is well known for contributing resistance to drugs and fungicides in fungi, and includes Pdr5p and Pdr10p from S. cerevisiae and Cdr1p, Cdr2p, Cdr3p and Cdr4p proteins from C. albicans. While functions of the subgroup I of ABC-G proteins has been investigated thoroughly, information about the subgroup V of fungal ABC-G is limited, including their biological roles. The similar expression patterns of the two $\mathrm{ABC}$ transporters with that of zhd101 suggests that these $C$. rosea $\mathrm{ABC}$ transporters evolved as a specific mechanism to withstand ZEA, potentially by providing efflux of ZEA and/or its digested products.

\section{Conclusions}

In conclusion, our SSH results suggested that tolerance to DON in C. rosea is provided by a consort of enzymes and proteins, covering a broad range of genes from metabolism 
to transporters. Cellular energy is manipulated to generate proteins to compensate for those that are destroyed by DON. This is ascertained by the increase in transcripts encoding (I) metabolic-related enzymes such as CYP450 and COX, (II) sugar transports such as HXT2 and $\mathrm{H}^{+}$-ATPase and (III) cellular response such as Hsp70 and Hsp90. On the other hand, two $\mathrm{ABC}$ transporters may participate in conferring resistance to ZEA together with ZHD101. This is the first time that participation of $A B C$ transporters in ZEA detoxification are implicated, which was thought previously to rely only on ZHD101.

\section{Methods}

\section{Fungal cultures}

C. rosea strain $\mathrm{IK} 726$ was revived from $-80^{\circ} \mathrm{C}$ glycerol stock on Czapek-Dox agar (Merck) for 5 days at room temperature. A plug of actively growing mycelium was subsequently transferred to $25 \mathrm{ml}$ Czepak-Dox broth (Sigma) in $250 \mathrm{ml}$ Erlenmeyer flask and incubated at $25^{\circ} \mathrm{C}$ for 5 days prior to toxin treatment.

Pure DON (cat. no. D0156) and ZEA (cat. no. Z2125) were purchased from Sigma-Aldrich and dissolved in methanol before storing at $-20^{\circ} \mathrm{C}$ as a stock. DON- or ZEA-containing methanol was applied separately into the culture medium to achieve a final concentration of 5 and 10 ppm, respectively. An equal amount of methanol was incorporated in the control experiment. After the treatments, the cultures were incubated for 72 and 2 hours for DON and ZEA treatment, respectively, at $25^{\circ} \mathrm{C}$ on a $150 \mathrm{rpm}$ rotary shaker before harvesting mycelium by vacuum filtration. The harvested mycelium was flash frozen with liquid nitrogen and stored at $-80^{\circ} \mathrm{C}$ until use.

\section{RNA extraction and construction of DON- and ZEA-induced subtractive cDNA libraries}

Total RNA was extracted from DON-, ZEA-treated and control samples using Spectrum ${ }^{\mathrm{Tm}}$ Plant total RNA kit (Sigma) according to the manufacturer's protocol. To ensure the absence of DNA impurities, removal of residual DNA was achieved by on-column DNA digestion RNaseFree DNase Set (Qiagen) following the manufacturer's protocol. The RNA obtained was quantified and monitored for quality by Nanodrop spectrophotometer ND-1000 (Thermo Scientific). Subsequently, mRNA was extracted from $100 \mu \mathrm{g}$ total RNA by Dynabeads ${ }^{\oplus}$ mRNA Purification Kit (Invitrogen) before proceeding with subtractive hybridization.

750 ng mRNA from DON-, ZEA-treated and control samples was used to generate each subtractive cDNA library. Synthesis of double-stranded cDNA for all treatments and suppression subtractive hybridization (SSH) utilised PCR select ${ }^{\mathrm{TM}}$ Subtractive Hybridization Kit (Clontech) according to the manufacturer's protocol. Only forward subtraction was performed with DON- or ZEA-treated
mRNA as the driver and control treatment mRNA as the tester for each library.

Amplification of the subtracted transcripts was performed using Advantage Taq polymerase (Clontech). A $2 \mu \mathrm{l}$ aliquot of the PCR product obtained from each library were cloned into the $\mathrm{pCR}^{\circ} \mathrm{II}-\mathrm{TOPO}^{\circ}$ vector using TOPO TA cloning kit (Invitrogen) before subsequent transformation to Library Efficiency ${ }^{\circ} \mathrm{DH} 5 \alpha^{\mathrm{Tm}}$ chemical competent cells (Invitrogen). Colony PCR of a total of 480 randomly picked clones from DON- and ZEA-subtracted cDNA libraries was performed using M13 primers and Hotmaster $^{\oplus}$ Taq DNA Polymerase (5 PRIME) on Gene Amp PCR system 2400 (Applied BioSystem). The PCR products were purified using QIAquick ${ }^{\circledR}$ PCR purification kit (Qiagen) according to the manufacturer's protocol and were subject to gel electrophoresis with $1 \%$ agarose. PCR products larger than 200 base pairs were collected and sequenced using Applied Biosystems 3730XL Sanger sequencing with BigDye terminator serviced by Beckman Coulter genomics (Essex, United Kingdom).

\section{Sequence analysis and annotation}

A total of 480 sequences acquired from each library were cleansed and trimmed to remove a vector backbone and assembled using the software package CLC Main Workbench version 6.5 (CLC Bio, Denmark). BLASTX [43] was adopted to search for similar non-redundant proteins in GenBank protein database [44] using the BLAST function of CLC Main Workbench with the cut-off E-value of $10^{-6}$. Sequences with no significant hit from BLASTX were subjected to BLASTN against $\mathrm{nr} / \mathrm{nt}$ nucleotide collection of the GenBank with the cut-off E-value of $10^{-6}$. Functional annotation of ESTs with significant database matches was performed using BLAST2GO where the Gene Ontology (GO) annotation of level 2 biological process was achieved [45]. The GO annotation was analysed using default settings with and E-value threshold $=10^{-6}$.

\section{Quantitative reverse transcription - polymerase chain reaction (qRT-PCR) analysis for validation of SSH results} To validate genes up-regulated during exposure to DON or ZEN, qRT-PCR was performed with five genes from each library selected for their putative involvement in secondary metabolite resistance or because of their high level of EST redundancy. Fungal culture and inoculation of the toxins were carried out in triplicate as a separate experiment as mentioned above except that fungal mycelia were collected temporally at 2, 6, 12, 36 and 72 hours after inoculation (hai). The mycelia were immediately flash frozen with liquid nitrogen and were kept at $-80^{\circ} \mathrm{C}$ until use.

Total RNA extraction was conducted using RNeasy Plant Mini Kit (Qiagen) prior to RiboLock RNase inhibitor (Fermentas) and DNase I (Fermentas) treatment to remove DNA contaminants following the manufacture's protocol. 
Table 3 List of primers used in the qRT-PCR validation

\begin{tabular}{lll}
\hline Target gene & Forward primer & Reverse primer \\
\hline DON-induced library & & ACAGCGCGAGGAACATACTT \\
$\beta$-tubulin & GGTCAGTGCGGTAACCAAAT & CTCGCTTGGGCTGTGAAT \\
Hexose transporter-like protein & GCGCAACATCCGGCAAAAA & ATGTTCTGGGTGTGCAT \\
CYP50 NOR & CTTGTGTTGAGCAGCTT & ACAACCCACCCCGATTATA \\
This/Pfpl family protein & ATTCTCATCCTCGTCACCC & GAAGCTACACAGGACGCA \\
Diacylglycerol o-acyltransferase & AGCGTCAATAAGGTGTGG & GTGTCCCAGATGCCAAAGT \\
Pyruvate decarboxylase & CCCAACCAAGTCCATCTGT & \\
ZEA-induced library & & ACAGCGCGAGGAACATACTT \\
$\beta$-tubulin & GGTCAGTGCGGTAACCAAAT & CGCCTCCGAGCCTCCAGACAC \\
Zearalenone hydrolase & GTGCCACGAACTGCCAACAAAG & GGTATTTGCTCTGCCTCTG \\
abcG29 & CAGCCCCGAGTTAGCAA & CCTCACTGTTCTCCAGC \\
abcG5 & GTCAACTTGGGTTCGAATG & ACTCCGAGGAAGAATCGC \\
Major facilitator transporter & ATCCCATTACCAACGCCA & ACGAAGATCAGCCAGTCC \\
Glycoside hydrolase family 5 & CGCACGTGAACAATCCTC &
\end{tabular}

The total RNA was quantified with Nanodrop spectrophotometer (Thermo Scientific). First-strand cDNA was synthesised from $1 \mu \mathrm{g}$ total RNA using iScript cDNA synthesis kit (BIO-RAD) with random primers according to the manufacturer's protocol. Gene expression analysis was performed in two technical replicates for each biological replicate on a Mx3000P qPCR system (Stratagene) with $150 \mathrm{ng}$ of cDNA and Maxima ${ }^{\circledR}$ SYBR Green qPCR Master Mix (Fermentas). A selected set of gene-specific primers for DON- and ZEA-induced cDNA libraries was listed in Table 3. Analysis of melting curves was carried out at the end of each run to evaluate undesired amplifications. Expression of tub2 gene encoding $\beta$-tubulin that was previously evaluated to be constitutively and constantly expressed in C. rosea [46] was used as a housekeeping gene to normalise target gene data. Subsequently, temporal expression of each selected genes at all time points were compared to its expression at 2 hours using the $2^{-\Delta \Delta} \mathrm{CT}$ relative gene expression method [47]. This was done to achieve an overview of gene expression dynamics in comparison to the earliest response at 2 hours. Gene expression data were analysed statistically using analysis of variance (ANOVA) with a General Linear Model implemented in MINITAB version 15 (Minitab Inc.). Pairwise comparisons were made using Tukey's method with a confidential level of $95 \%$.

\section{Bioinformatics prediction of the ZEA-induced full-length $A B C$ transporters}

Full-length sequences of ZEA-induced ABC transporters were predicted from an Illumina- and SOLiD-based draft genome assembly of the C. rosea IK726 genome (Karlsson et al., unpublished) using FGENESH + [48] and a F. graminearum ABC transporter (locus FGSG_03735) as the template. To categorize the $\mathrm{ABC}$ transporters, amino acid sequences of known fungal $\mathrm{ABC}$ transporters from $F$. graminearum, A. nidulans and Neurospora crassa [25] were used as references to generate a phylogenetic tree. Sequences were aligned by ClustalW [49] implemented in the Molecular Evolution and Genetics Analysis (MEGA) software package version 5 [50]. Phylogenetic analyses were performed using Neighbour-Joining implemented in MEGA5, using pairwise deletion of gaps and the Poisson correction distance of substitution rates. Statistical support for phylogenetic grouping was estimated by 1000 bootstrap resamplings.

\section{Availability of supporting data}

The data sets supporting the results of this article are included within the article and its additional files.

\section{Additional files}

Additional file 1: DON-induced library.

Additional file 2: ZEA-induced library.

Additional file 3: Predicted ABC transporters.

Competing interests

The authors declare that they have no competing interests.

Authors' contributions

CK performed the experiments, bioinformatics and transcriptome analyses, participated in the design of the study and drafted the manuscript. MK performed bioinformatics analysis, participated in the design of the study and the draft manuscript. AD participated in transcriptome analysis and drafted the manuscript. DFJ and DBC conceived the study, participated in its design and coordination. All authors have contributed to writing the manuscript. All authors read and approved the final manuscript. 


\section{Acknowledgement}

The Danish research council and Swedish University of Agriculture Sciences are thanked for Ph.D. studentship to CK via the Research program for Biotechnology (FOBI) at the University of Copenhagen.

Received: 21 August 2013 Accepted: 16 January 2014 Published: 22 January 2014

\section{References}

1. Nielsen LK, Jensen JD, Nielsen GC, Jensen JE, Spliid NH, Thomsen IK, Justesen AF, Collinge DB, Jørgensen LN: Fusarium head blight of cereals in Denmark: species complex and related mycotoxins. Phytopathology 2011, 101:960-969.

2. Desjardins AE: Fusarium Mycotoxins : Chemistry, Genetics and Biology. St. Paul, MN: APS Press, American Phytopathological Society; 2006

3. Jansen C, von Wettstein D, Schafer W, Kogel KH, Felk A, Maier FJ: Infection patterns in barley and wheat spikes inoculated with wild-type and trichodiene synthase gene disrupted Fusarium graminearum. Proc Natl Acad Sci USA 2005, 102:16892-16897.

4. Reddy K, Salleh B, Saad B, Abbas H, Abel C, Shier W: An overview of mycotoxin contamination in foods and its implications for human health. Toxin Rev 2010, 29:3-26.

5. Pestka JJ: Deoxynivalenol: mechanisms of action, human exposure, and toxicological relevance. Arch Toxicol 2010, 84:663-679.

6. Lutz MP, Feichtinger G, Defago G, Duffy B: Mycotoxigenic Fusarium and deoxynivalenol production repress chitinase gene expression in the biocontrol agent Trichoderma atroviride P1. Appl Environ Microbiol 2003, 69:3077-3084

7. Caldwell RW, Tuite J, Stob M, Baldwin R: Zearalenone production by Fusarium species. Appl Microbiol 1970, 20:31-34

8. Zinedine A, Soriano JM, Molto JC, Manes J: Review on the toxicity, occurrence, metabolism, detoxification, regulations and intake of zearalenone: an oestrogenic mycotoxin. Food Chem Toxicol 2007, 45:1-18.

9. Lysoe $\mathrm{E}$, Bone KR, Klemsdal SS: Real-time quantitative expression studies of the zearalenone biosynthetic gene cluster in Fusarium graminearum. Phytopathology 2009, 99:176-184.

10. Utermark J, Karlovsky P: Role of zearalenone lactonase in protection of Gliocladium roseum from fungitoxic effects of the mycotoxin zearalenone. Appl Environ Microbiol 2007, 73:637-642.

11. Jard G, Liboz T, Mathieu F, Guyonvarc'h A, André F, Delaforge M, Lebrihi A: Transformation of zearalenone to zearalenone-sulfate by Aspergillus spp. World Mycotoxin J 2010, 3:183-191.

12. Kakeya H, Takahashi-Ando N, Kimura M, Onose R, Yamaguchi I, Osada H: Biotransformation of the mycotoxin, zearalenone, to a non-estrogenic compound by a fungal strain of Clonostachys sp. Biosci Biotechnol Biochem 2002, 66:2723-2726

13. Takahashi-Ando N, Kimura M, Kakeya H, Osada H, Yamaguchi I: A novel lactonohydrolase responsible for the detoxification of zearalenone: enzyme purification and gene cloning. Biochem J 2002, 365:1-6.

14. el-Sharkawy S, Abul-Hajj YJ: Microbial cleavage of zearalenone. Xenobiotica 1988, 18:365-371.

15. Kriszt R, Krifaton C, Szoboszlay S, Cserháti M, Kriszt B, Kukolya J, Czéh Á Fehér-Tóth S, Török L, Szőke Z, et al: A new zearalenone biodegradation strategy using non-pathogenic Rhodococcus pyridinivorans K408 strain. PLoS One 2012, 7:e43608

16. Garda-Buffon J, Badiale-Furlong E: Kinetics of deoxynivalenol degradation by Aspergillus oryzae and Rhizopus oryzae in submerged fermentation. J Braz Chem Soc 2010, 21:710-714.

17. He C, Fan Y, Liu G, Zhang H: Isolation and identification of a strain of Aspergillus tubingensis with deoxynivalenol biotransformation capability. Int J Mol Sci 2008, 9:2366-2375.

18. Franco TS, Garcia S, Hirooka EY, Ono YS, dos Santos JS: Lactic acid bacteria in the inhibition of Fusarium graminearum and deoxynivalenol detoxification. J App/ Microbiol 2011, 111:739-748.

19. Karlovsky P: Biological detoxification of the mycotoxin deoxynivalenol and its use in genetically engineered crops and feed additives. Appl Microbiol Biotechnol 2011, 91:491-504.

20. Li XZ, Zhu C, de Lange CF, Zhou T, He J, Yu H, Gong J, Young JC: Efficacy of detoxification of deoxynivalenol-contaminated corn by Bacillus sp. LS100 in reducing the adverse effects of the mycotoxin on swine growth performance. Food Addit Contam Part A Chem Anal Control Expo Risk Assess 2011, 1:1-8.
21. Guan S, He J, Young JC, Zhu H, Li X-Z, Ji C, Zhou T: Transformation of trichothecene mycotoxins by microorganisms from fish digesta. Aquaculture 2009, 290:290-295.

22. Jensen B, Knudsen IB, Jensen D: Biological seed treatment of cereals with fresh and long-term stored formulations of Clonostachys rosea: biocontrol efficacy against Fusarium culmorum. Eur J Plant Pathol 2000 106:233-242.

23. Jensen $B$, Knudsen IM, Madsen M, Jensen DF: Biopriming of infected carrot seed with an antagonist, Clonostachys rosea, selected for control of seedborne Alternaria spp. Phytopathology 2004, 94:551-560.

24. Knudsen IMB, Hockenhull J, Jensen DF: Biocontrol of seedling diseases of barley and wheat caused by Fusarium culmorum and Bipolaris sorokiniana: effects of selected fungal antagonists on growth and yield components. Plant Pathol 1995, 44:467-477.

25. Kovalchuk A, Driessen AJ: Phylogenetic analysis of fungal $A B C$ transporters. BMC Genomics 2010, 11:177.

26. Stephens C, Harrison S, Kazan K, Smith FN, Goulter K, Maclean D, Manners J: Altered fungal sensitivity to a plant antimicrobial peptide through over-expression of yeast cDNAs. Curr Genet 2005, 47:194-201.

27. Herrero S, Amnuaykanjanasin A, Daub ME: Identification of genes differentially expressed in the phytopathogenic fungus Cercospora nicotianae between cercosporin toxin-resistant and -susceptible strains. FEMS Microbiol Lett 2007, 275:326-337.

28. Lewis DA, Bisson LF: The Hxt1 gene-product of Saccharomyces cerevisiae is a new member of the family of hexose transporters. Mol Cell Biol 1991, 11:3804-3813.

29. Struck $\mathrm{C}$, Hahn M, Mendgen K: Plasma membrane $\mathrm{H}^{+}$-ATPase activity in spores, germ tubes, and haustoria of the rust fungus Uromyces viciae-fabae. Fungal Genet Biol 1996, 20:30-35.

30. Struck C, Siebels C, Rommel O, Wernitz M, Hahn M: The plasma membrane $\mathrm{H}^{+}$-ATPase from the biotrophic rust fungus Uromyces fabae: molecular characterization of the gene (PMA1) and functional expression of the enzyme in yeast. Mol Plant Microbe Interact 1998, 11:458-465.

31. Portillo F: Regulation of plasma membrane $\mathrm{H}^{+}$-ATPase in fungi and plants. Biochim Biophys Acta 2000, 1469:31-42

32. Zhang $X$, Jiang $L$, Geng $C$, Cao J, Zhong L: The role of oxidative stress in deoxynivalenol-induced DNA damage in HepG2 cells. Toxicon 2009, 54:513-518

33. Parsell DA, Lindquist S: The function of heat-shock proteins in stress tolerance: degradation and reactivation of damaged proteins. Annu Rev Genet 1993, 27:437-496.

34. Chen $Y$, Sanchez $Y$ : Chk1 in the DNA damage response: conserved roles from yeasts to mammals. DNA Repair (Amst) 2004, 3:1025-1032.

35. Perez-Martin J: DNA-damage response in the basidiomycete fungus Ustilago maydis relies in a sole Chk1-like kinase. DNA Repair (Amst) 2009, 8:720-731.

36. Cowen LE: Hsp90 orchestrates stress response signaling governing fungal drug resistance. PLOS Pathog 2009, 5:e1000471.

37. Singh SD, Robbins N, Zaas AK, Schell WA, Perfect JR, Cowen LE: Hsp90 governs echinocandin resistance in the pathogenic yeast Candida albicans via calcineurin. PLoS Pathog 2009, 5:e1000532

38. Kimura T, Komiyama T, Furuichi Y, limura Y, Karita S, Sakka K, Ohmiya K: $\mathrm{N}$-glycosylation is involved in the sensitivity of Saccharomyces cerevisiae to HM-1 killer toxin secreted from Hansenula mrakii IFO 0895. Appl Microbiol Biotechnol 1999, 51:176-184.

39. Maddi A, Fu C, Free SJ: The Neurospora crassa dfg5 and dcw1 genes encode $a-1,6-$ mannanases that function in the incorporation of glycoproteins into the cell wall. PLoS One 2012, 7:e38872.

40. Gentzsch M, Strahl-Bolsinger S, Tanner W: A new Dol-P-Man:protein O-D-mannosyltransferase activity from Saccharomyces cerevisiae. Glycobiology 1995, 5:77-82.

41. Bouzarelou D, Billini M, Roumelioti K, Sophianopoulou V: EgID, a putative endoglucanase, with an expansin like domain is localized in the conidial cell wall of Aspergillus nidulans. Fungal Genet Biol 2008, 45:839-850.

42. Cannon RD, Lamping E, Holmes AR, Niimi K, Baret PV, Keniya MV, Tanabe K, Niimi M, Goffeau A, Monk BC: Efflux-mediated antifungal drug resistance. Clin Microbiol Rev 2009, 22:291-312.

43. Altschul SF, Madden TL, Schaffer AA, Zhang J, Zhang Z, Miller W, Lipman DJ: Gapped BLAST and PSI-BLAST: a new generation of protein database search programs. Nucleic Acids Res 1997, 25:3389-3402.

44. Benson DA, Karsch-Mizrachi I, Lipman DJ, Ostell J, Wheeler DL: GenBank Nucleic Acids Res 2008, 36:D25-D30. 
45. Conesa A, Gotz S, Garcia-Gomez JM, Terol J, Talon M, Robles M: Blast2GO: a universal tool for annotation, visualization and analysis in functional genomics research. Bioinformatics 2005, 21:3674-3676.

46. Mamarabadi $M$, Jensen $B$, Jensen DF, Lübeck M: Real-time RT-PCR expression analysis of chitinase and endoglucanase genes in the three-way interaction between the biocontrol strain Clonostachys rosea IK726, Botrytis cinerea and strawberry. FEMS Microbiol Lett 2008, 285:101-110.

47. Livak KJ, Schmittgen TD: Analysis of relative gene expression data using real-time quantitative PCR and the $2^{-\Delta \Delta C t}$ method. Methods 2001 25:402-408.

48. Solovyev V, Kosarev P, Seledsov I, Vorobyev D: Automatic annotation of eukaryotic genes, pseudogenes and promoters. Genome Biol 2006, 7(Suppl 1):S10.1-S10.12.

49. Larkin MA, Blackshields G, Brown NP, Chenna R, McGettigan PA, McWilliam H, Valentin F, Wallace IM, Wilm A, Lopez R, et al: Clustal W and Clustal X version 2.0. Bioinformatics 2007, 23:2947-2948.

50. Tamura K, Peterson D, Peterson N, Stecher G, Nei M, Kumar S: MEGA5: molecular evolutionary genetics analysis using maximum likelihood, evolutionary distance, and maximum parsimony methods. Mol Biol Evol 2011, 28:2731-2739.

doi:10.1186/1471-2164-15-55

Cite this article as: Kosawang et al:: Transcriptomic profiling to identify genes involved in Fusarium mycotoxin Deoxynivalenol and Zearalenone tolerance in the mycoparasitic fungus Clonostachys rosea. BMC Genomics 2014 15:55.

\section{Submit your next manuscript to BioMed Central and take full advantage of:}

- Convenient online submission

- Thorough peer review

- No space constraints or color figure charges

- Immediate publication on acceptance

- Inclusion in PubMed, CAS, Scopus and Google Scholar

- Research which is freely available for redistribution 